\title{
Load Speed Regulation in Compliant Mechanical Transmission Systems Using Feedback and Feedforward Control Actions *
}

\author{
P. R. Raul ${ }^{\text {a }}$, R. V. Dwivedula ${ }^{\mathrm{b}}$, P. R. Pagilla ${ }^{\text {a }}$ \\ ${ }^{a}$ Mechanical 8 Aerospace Engineering, Oklahoma State University, Stillwater, \\ OK, 74078 \\ ${ }^{\mathrm{b}}$ Department of Mechanical Engineering, Sree Vidyanikethan Engineering College, \\ Tirupati, Andhra Pradesh, 517102, India
}

^ Corresponding author P. R. Pagilla. Tel. +1-405-744-6579. Fax +1-405-744-7873. Email addresses: pramod.raul@okstate.edu (P. R. Raul), ramamurthy@vidyanikethan.edu (R. V. Dwivedula), pagilla@okstate.edu (P. R. Pagilla). 


\begin{abstract}
In this work we consider the problem of controlling the load speed of a mechanical transmission system consisting of a belt-pulley and a gear-pair to transmit mechanical power from the motor shaft to the load shaft. The system is modeled as two inertias (motor and load) connected by a compliant transmission system. In practice, control systems using either motor speed feedback or load speed feedback have been employed. If the transmission is assumed to be rigid, then using either the motor or load speed feedback provides the same result. In the presence of transmission compliance, which may be due to belt compliance or torsional compliance of long shafts, the stability and performance characteristics of the closed-loop system are quite different based on whether motor speed or load speed feedback are employed. In this paper we investigate both the motor and load speed feedback options by considering transmission compliance and utilizing the singular perturbation method. We also propose and discuss a control scheme that utilizes both motor and load speed feedback. An adaptive feedforward action is employed for this control scheme to reject torque disturbances on the load. We consider an experimental platform of such a system for conducting experiments that is typically utilized in roll-to-roll manufacturing. Representative experimental results are shown and discussed.
\end{abstract}

Key words: Adaptive feedforward, load speed regulation, belt-pulley mechanical transmission.

\title{
1 Introduction
}

Mechanical transmissions are widely used in various industries where the mechanical power is typically transmitted from motor shafts to load shafts by utilizing transmission systems. Examples include manufacturing, power generation, and transportation systems. Power transmission with speed reduction and variable torque requirement is made possible with mechanical transmission systems. Belt-pulley and gear transmission systems are commonly used. In many applications, a mechanical transmission system containing a combination of belt-pulley and a gear-pair is very convenient over a purely gear transmission system. When the center distance between the driving (motor) shaft and the driven (load) shaft is too large for use of a single gear-pair, using a belt to transmit motion/power may be the only practical alternative. Further, such an arrangement is advantageous because coupling the drive motor directly to the process end mandates very accurate collinearity of the axes and takes considerable amount of time; also, there is no guarantee that collinearity is maintained over extended period of time due to load disturbances. Belt driven transmission systems offer considerable flexibility as small inaccuracies in alignment can be absorbed into compliance of the belt. However, compliance 
of the belt introduces additional dynamics into the system. The belt driven power transmission system is common in roll to roll manufacturing. The presence of compliance from transmissions and the stiffness of web material [1] will pose different levels of severity in properly transporting the web.

Control of load speed is essential in many applications. When rigid transmissions are employed, there is no dynamic relation between the motor shaft and the load shaft, and typically the motor shaft speed is controlled to control the speed of the load shaft. However, due to the transmission dynamics, resulting from the compliance of belt as well as long shafts in the transmission, regulating load shaft speed is not the same as regulating motor shaft speed. In the presence of such a transmission, practicing engineers are often confronted with the question of whether to use (i) motor speed feedback to control load speed as is done in conventional practice, or (ii) use load speed feedback, or (iii) use a combination of motor and load speed feedback.

There is a large body of literature on the characteristics of belt drives and design of mechanisms using belt drives. Much of this work focused on the mechanism of motion/power transfer, location and extent of slip-arc, nature of frictional contact, efficiency limit of the belt-drive system, and methodology of design/selection of belt-drive components [2-11]. In [12], modeling and control of a belt-drive positioning table is discussed, and in [13], direct drive control of $\mathrm{X}-\mathrm{Y}$ table is presented. However, no specific model is reported for including the effect of compliance of the belt; system identification techniques were used to obtain the system dynamics, to be later used in tuning of the feedback gains. Similarly in [14], a composite fuzzy controller, consisting of a feedback fuzzy controller and a feed-forward acceleration compensator, is proposed to control a belt drive precision positioning table; the effects of belt compliance were not included in this paper. In [15], a robust motion control algorithm for beltdriven servomechanism is reported. In this paper, the belt-stretch dynamics is assumed to contribute a pair of purely imaginary poles to the transfer function of the system; the fact that the belt serves as an interconnection from loadside to the motor-side is ignored in this paper. Modeling of belt-pulley and gear-pair transmission system with gear backlash is given in [16]. Analysis and control of speed drive systems with torsional loads is reported in [17-20].

The following are the contributions of this work: Based on the model of the two inertias (motor and load) connected by a belt-pulley and gear-pair transmission system, we have investigated the effect of using either motor or load feedback to control the load speed by utilizing the singular perturbation method. In each case, we consider a PI controller that is typical in the industry for the feedback controller. The small parameter in the singular perturbation method is proportional to the reciprocal of the square root of the belt compliance. The singular perturbation analysis revealed that the controller using pure load feedback results in an unstable system. Therefore, use of pure load feedback 
must be avoided. To directly control the load speed, we also propose a control scheme that uses both the motor speed and load speed feedback and show that such scheme results in a stable closed-loop system. Since feedback action is not sufficient in rejecting periodic disturbances that commonly act on the load, we also consider an adaptive feedforward compensation action that is based on adaptive estimation of the coefficients of the periodic disturbance as suggested in [21]. This adaptive feedforward action is quite suitable for this application because it preserves closed-loop stability achieved with the feedback controller. Experiments were conducted to evaluate the performance of the various control schemes on an industrial grade transmission system that is common in roll-to-roll manufacturing.

The remainder of the paper is organized as follows. The model of the system is described in Section 2. Sections 3 and 4 describe the motor speed feedback only and load speed feedback only cases, respectively. A control scheme that utilizes both motor and load speed feedback is discussed in Section 5. An add-on adaptive feedforward compensation to reject load speed disturbances is discussed in Section 6. Section 7 provides a description of the experimental platform and a comparison of the results with the various control schemes. Conclusions are given in Section 8.

\section{Model of the System}

A schematic of the belt-pulley and gear transmission system connecting the motor with the load is shown in Figure 1 . In the schematic, $J_{i}$ denote the inertias, $b_{i}$ denote the viscous friction coefficients, $R_{i}$ denote the radii of the pulleys and gears, $\theta_{i}$ denote the angular displacements of the inertias, $\tau_{m}$ denotes the motor torque, $\tau_{L}$ denotes the torque disturbance on the load, and $K_{b}$ denotes the stiffness of the belt.

To derive the governing equations for this system we consider the action of the belt in transmitting power. For a given direction of rotation of the pulley, the belt has a tight side and a slack side as shown in Figure 1. It is assumed that the transmission of power is taking place on the tight side and the transport of the belt is taking place on the slack side. Under this assumption, the net change in tension on the slack side will be much smaller than that in the tight side and thus may be ignored. The tight side of the belt can then be modeled as a spring with spring constant of $K_{b}$. For given angular displacements $\theta_{m}$ and $\theta_{L}$, the net elongation of the tight side of the belt can be written as $\left(R_{1} \theta_{m}-G_{R} R_{2} \theta_{L}\right)$. Because of this elongation, the driving pulley experiences a torque of $\left(R_{1} \theta_{m}-G_{R} R_{2} \theta_{L}\right) K_{b} R_{1}$ and the driven pulley experiences a torque of $\left(R_{1} \theta_{m}-G_{R} R_{2} \theta_{L}\right) G_{R} R_{2} K_{b}$. Under the assumption that the inertias of the pulleys and gears are much smaller than the motor and the load, the governing 


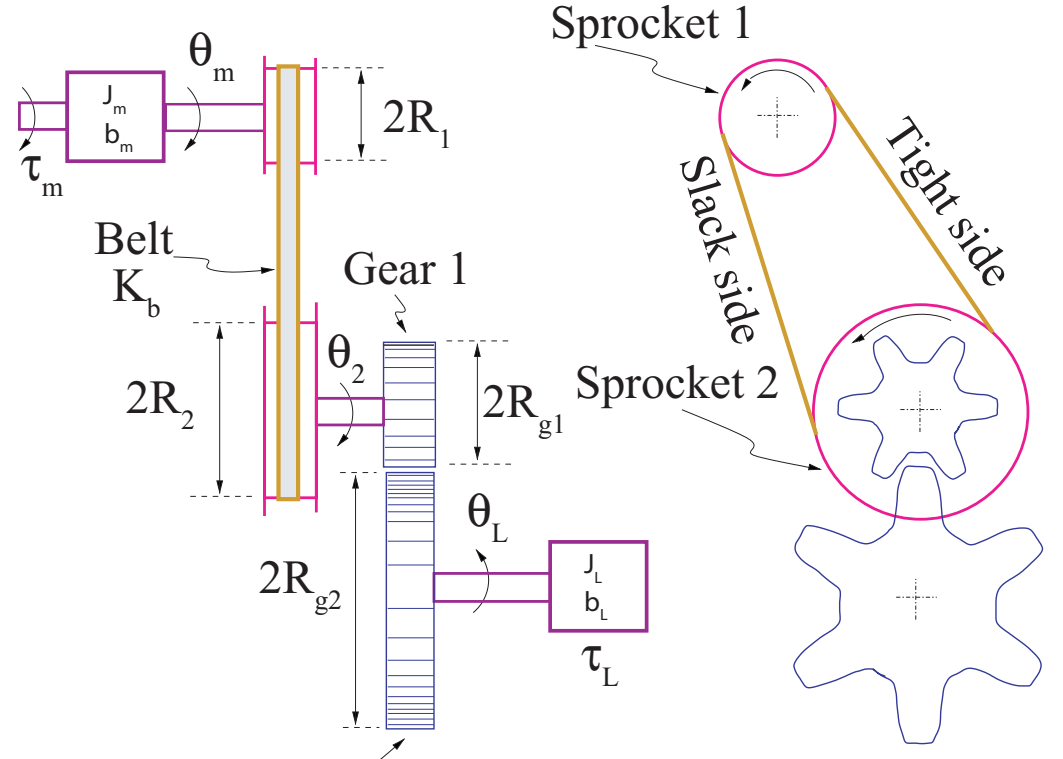

Gear 2

Fig. 1. Schematic of a belt-pulley and gear-pair transmission system

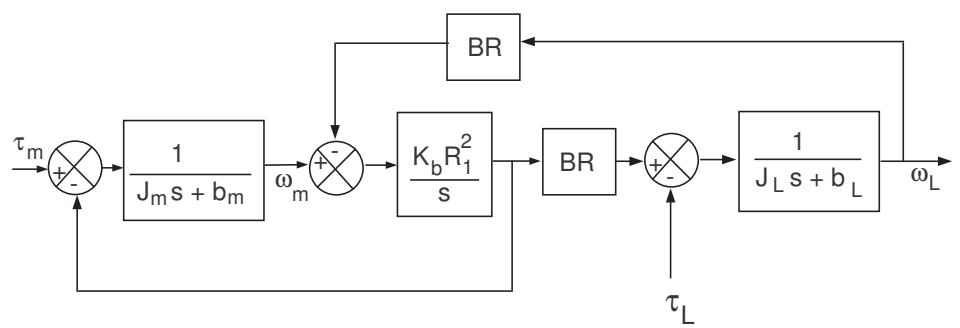

Fig. 2. Block diagram of the belt-pulley and gear transmission system; $B R$ denotes the overall speed ratio, $B R=\left(R_{2} / R_{1}\right) G_{R}$.

equations of motion for the motor-side inertia and the load-side inertia are given by

$$
\begin{aligned}
J_{m} \ddot{\theta}_{m}+b_{m} \dot{\theta}_{m}+R_{1} K_{b}\left(R_{1} \theta_{m}-G_{R} R_{2} \theta_{L}\right) & =\tau_{m}, \\
J_{L} \ddot{\theta}_{L}+b_{L} \dot{\theta}_{L}-G_{R} R_{2} K_{b}\left(R_{1} \theta_{m}-G_{R} R_{2} \theta_{L}\right) & =\tau_{L} .
\end{aligned}
$$

A block diagram representation of the system given by (1) is provided in Figure 2 ; note that this block diagram represents the open-loop system and the two "loops" appearing in the block diagram that represent the interconnections in (1). The open-loop transfer functions from the motor torque signal $\tau_{m}$ to the 
motor speed $\omega_{m}$ and load speed $\omega_{L}$ are given by

$$
\begin{aligned}
& G_{\tau_{m} \omega_{m}}(s) \triangleq \frac{\omega_{m}(s)}{\tau_{m}(s)}=\frac{J_{L} s^{2}+b_{L} s+G_{R}^{2} R_{2}^{2} K_{b}}{D(s)}, \\
& G_{\tau_{m} \omega_{L}}(s) \triangleq \frac{\omega_{L}(s)}{\tau_{m}(s)}=\frac{G_{R} R_{1} R_{2} K_{b}}{D(s)},
\end{aligned}
$$

where

$$
\begin{aligned}
D(s) & =J_{m} J_{L} s^{3}+\left(b_{L} J_{m}+J_{L} b_{m}\right) s^{2}+\left(K_{b} J_{e q}+b_{m} b_{L}\right) s \\
& +K_{b} b_{e q} \\
J_{e q} & =G_{R}^{2} R_{2}^{2} J_{m}+R_{1}^{2} J_{L}, \\
b_{e q} & =G_{R}^{2} R_{2}^{2} b_{m}+R_{1}^{2} b_{L} .
\end{aligned}
$$

The goal is to control load speed. In the following we will discuss the closedloop control systems that consider three scenarios: (i) pure motor speed feedback or (ii) pure load speed feedback or (iii) a combination of motor and load speed feedback.

\section{Motor Speed Feedback Control Scheme}

It is common to control load speed by using measurement of motor speed $\omega_{m}$ as feedback. This control scheme is shown in Figure 3. The control structure is designed to regulate motor speed $\omega_{m}$ to the reference $\omega_{r m}$, and thereby indirectly regulate load speed $\omega_{L}$.

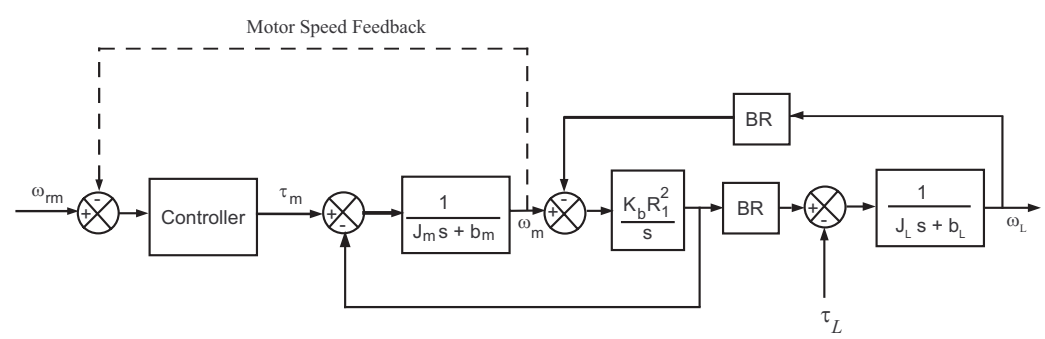

Fig. 3. Motor speed feedback control scheme

We consider the often used Proportional-Integral (PI) control action which is widely used in industrial environments. The feedback law is given by

$$
\tau_{m}=K_{p m}\left(\omega_{r m}-\omega_{m}\right)+K_{i m} \int\left(\omega_{r m}-\omega_{m}\right) d \tau
$$

With this control law, the closed-loop transfer function from $\omega_{r m}$ to $\omega_{L}$ is obtained as 


$$
\frac{\omega_{L}(s)}{\omega_{r m}(s)}=\frac{\left(G_{R} R_{1} R_{2} K_{b} / J_{m} J_{L}\right)\left(s K_{p m}+K_{i m}\right)}{\psi_{m}(s)}
$$

where

$$
\begin{aligned}
\psi_{m}(s) & =s^{4}+c_{3} s^{3}+c_{2} s^{2}+c_{1} s+c_{0} \\
c_{3} & =\frac{\left(b_{m} J_{L}+J_{m} b_{L}+K_{p m} J_{L}\right)}{J_{m} J_{L}} \\
c_{2} & =\frac{\left(K_{b} J_{e q}+b_{m} b_{L}+K_{p m} b_{L}+K_{i m} J_{L}\right)}{J_{m} J_{L}} \\
c_{1} & =\frac{\left(K_{b} b_{e q}+G_{R}^{2} R_{2}^{2} K_{b} K_{p m}+K_{i m} b_{L}\right)}{J_{m} J_{L}} \\
c_{0} & =\frac{G_{R}^{2} R_{2}^{2} K_{b} K_{i m}}{J_{m} J_{L}}
\end{aligned}
$$

Note the the coefficients $c_{0}$ to $c_{3}$ depend on the controller gains. We consider the singular perturbation method for analyzing such a system with the small parameter proportional to the reciprocal of the square root of the belt stiffness $K_{b}$. For conduction singular perturbation analysis, we need to express the equations in the form

$$
\begin{aligned}
\dot{x} & =A_{11} x+A_{12} z, x\left(t_{0}\right)=x^{0} \\
\varepsilon \dot{z} & =A_{21} x+A_{22} z, z\left(t_{0}\right)=z^{0}
\end{aligned}
$$

where $x$ and $z$ are the states of the slow and the fast subsystems, respectively, and $\varepsilon$ is the small parameter; for our system we will consider $\varepsilon^{2}=1 / K_{b}$. The elements of matrices $A_{i j}$ may depend on $\varepsilon$. However, to use the singular perturbation method, the matrix $A_{22}$ needs to be nonsingular [22] at $\varepsilon=0$. A natural choice of the state variables for the singular perturbation analysis is $\theta_{m}, \dot{\theta}_{m}, \theta_{L}$ and $\dot{\theta}_{L}$. However, with this choice of the state variables, the matrix $A_{22}$ becomes singular at $\varepsilon=0$. To obtain a state-space representation in the form that would enable the use of the singular perturbation method, we consider the following transformation of variables:

$$
\begin{aligned}
& \theta_{c} \triangleq \frac{J_{m} \theta_{m}+J_{L} G_{R}\left(R_{2} / R_{1}\right) \theta_{L}}{J_{m}+J_{L}}, \\
& \theta_{s} \triangleq \theta_{m}-G_{R}\left(R_{2} / R_{1}\right) \theta_{L} .
\end{aligned}
$$

The variable $\theta_{c}$ is a weighted average of angular displacements $\left(\theta_{m}\right.$ and $\left.\theta_{L}\right)$ referred to the motor side and the variable $\theta_{s}$ is difference between the angular displacements $\left(\theta_{m}\right.$ and $\left.\theta_{L}\right)$ referred to the motor side; transformations similar to these have been used in prior studies of two inertia systems, see for example [23]. The idea of the weighted average of the displacements arises naturally in the case of a translational system wherein $\theta_{c}$ represents the position of the centroid of the masses. Now, choosing the state variables as $x=\left[\theta_{c}, \dot{\theta}_{c}\right]^{\top}$ and $z=\left[\theta_{s} / \varepsilon^{2}, \dot{\theta}_{s} / \varepsilon\right]^{\top}$, the state space representation of the system is obtained in 
the form given by (7) where

$$
\begin{gathered}
A_{11}=\left[\begin{array}{ll}
0 & 1 \\
f_{1} & f_{3}
\end{array}\right], A_{12}=\left[\begin{array}{cr}
0 & 0 \\
\varepsilon^{2} f_{21}+f_{22} & \varepsilon f_{4}
\end{array}\right], \\
A_{21}=\left[\begin{array}{ll}
0 & 0 \\
g_{1} & g_{3}
\end{array}\right], A_{22}=\left[\begin{array}{cr}
0 & 1 \\
\varepsilon^{2} g_{21}+g_{22} & \varepsilon g_{4}
\end{array}\right] \\
f_{1}=-K_{i m} / J_{0}, f_{21}=-K_{i m} J_{L} / J_{0}^{2}, \\
f_{22}=\left(G_{R}^{2} R_{2}^{2}-R_{1}^{2}\right) / J_{0}, f_{3}=-\left(K_{p m}+b_{m}+b_{L}\right) / J_{0}, \\
f_{4}=\left(b_{L} J_{m}-b_{m} J_{L}-K_{p m} J_{L}\right) / J_{0}^{2}, \\
g_{1}=-K_{i m} / J_{m}, g_{21}=-K_{i m} J_{L}^{2} /\left(J_{m} J_{L} J_{0}\right), \\
g_{22}=-\left(R_{1}^{2} J_{L}+G_{R}^{2} R_{2}^{2} J_{m}\right) /\left(J_{m} J_{L}\right), \\
g_{3}=\left(b_{L} J_{m}-b_{m} J_{L}-K_{p m} J_{L}\right) /\left(J_{m} J_{L}\right), \\
g_{4}=-\left(K_{p m} J_{L}^{2}+b_{m} J_{L}^{2}+b_{L} J_{m}^{2}\right) /\left(J_{m} J_{L} J_{0}\right),
\end{gathered}
$$

where $J_{0}=J_{m}+J_{L}$, and $1 / \varepsilon^{2}=K_{b}$. Notice that $\operatorname{det}\left(\left.A_{22}(\varepsilon)\right|_{\varepsilon=0}\right)=-g_{22} \neq 0$, thus satisfying the requirement of non-singularity of the matrix $A_{22}$ at $\varepsilon=0$. The characteristic equation for the system given by (9) can be factored as [22]

$$
\psi_{m}(s, \varepsilon) \approx \frac{1}{\varepsilon^{2}} \psi_{m s}(s, \varepsilon) \psi_{m f}(p, \varepsilon)=0
$$

with

$$
\begin{gathered}
\psi_{m s}(s, \varepsilon) \triangleq \operatorname{det}\left[s I_{2}-\left(A_{11}-A_{12} L(\varepsilon)\right)\right] \\
\psi_{m f}(p, \varepsilon) \triangleq \operatorname{det}\left[p I_{2}-\left(A_{22}+\varepsilon L(\varepsilon) A_{12}\right)\right]
\end{gathered}
$$

where $\psi_{m s}(s, \varepsilon)$ is the characteristic polynomial for the slow subsystem and $\psi_{m f}(p, \varepsilon)$ is the characteristic polynomial of the fast subsystem exhibited in the high-frequency scale $p=\varepsilon s$. The matrix $L(\varepsilon)$ is obtained using the iterative scheme given in [22].

Using the matrices given by equations (9), the slow and the fast characteristic polynomials are obtained as

$$
\begin{aligned}
\psi_{m s}(s, \varepsilon) & \approx s^{2}+\alpha_{1} s+\alpha_{0}, \\
\psi_{m f}(p, \varepsilon) & \approx p^{2}+\alpha_{1}^{\prime} p+\alpha_{0}^{\prime}
\end{aligned}
$$


where

$$
\begin{aligned}
\alpha_{1} & =\frac{G_{R}^{2} R_{2}^{2} b_{m}+R_{1}^{2} b_{L}+G_{R}^{2} R_{2}^{2} K_{p m}}{\left.G_{R}^{2} R_{2}^{2} J_{m}+R_{1}^{2} J_{L}\right)}, \\
\alpha_{0} & =\frac{G_{R}^{2} R_{2}^{2} K_{i m}}{G_{R}^{2} R_{2}^{2} J_{m}+R_{1}^{2} J_{L}} \\
\alpha_{1}^{\prime} & =\frac{G_{R}^{2} R_{2}^{2} K_{p m} J_{L}}{J_{m}\left(G_{R}^{2} R_{2}^{2} J_{m}+R_{1}^{2} J_{L}\right)} \varepsilon \\
\alpha_{0}^{\prime} & =\frac{G_{R}^{2} R_{2}^{2} J_{L}+R_{1}^{2} J_{m}}{J_{m} J_{L}} .
\end{aligned}
$$

Equation (12) indicates that both the fast and the slow subsystems are stable for all $K_{p m}, K_{i m}>0$. The result is true even without the approximation introduced by $L(\varepsilon)$ as shown in Theorem 2 in the Appendix.

\section{Load Speed Feedback Control Scheme}

One can employ the load speed feedback scheme shown in 4, where the measured variable is $\omega_{L}$. This seems to have the advantage of directly controlling load speed and attenuating the effect of the disturbance $\tau_{L}$. The feedback law

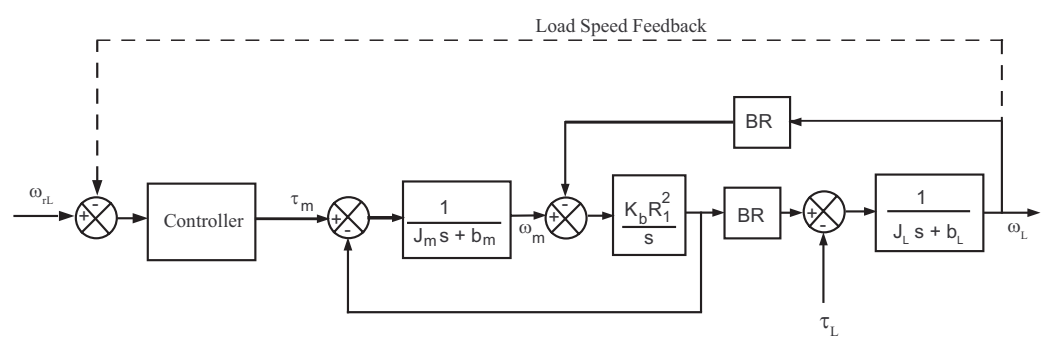

Fig. 4. Load speed feedback control scheme

is given by

$$
\tau_{m}=K_{p L}\left(\omega_{r L}-\omega_{L}\right)+K_{i L} \int\left(\omega_{r L}-\omega_{L}\right) d \tau
$$

and the closed-loop transfer function from $\omega_{r L}$ to $\omega_{L}$ is obtained as

$$
\frac{\omega_{L}(s)}{\omega_{r L}(s)}=\frac{\left(G_{R} R_{1} R_{2} K_{b} / J_{m} J_{L}\right)\left(s K_{p L}+K_{i L}\right)}{\psi_{L}(s)}
$$


where

$$
\begin{aligned}
\psi_{L}(s) & =s^{4}+d_{3} s^{3}+d_{2} s^{2}+d_{1} s+d_{0}, \\
d_{3} & =\frac{\left(b_{m} J_{L}+J_{m} b_{L}\right)}{J_{m} J_{L}}, \\
d_{2} & =\frac{\left(K_{b} J_{e q}+b_{m} b_{L}\right)}{J_{m} J_{L}} \\
d_{1} & =\frac{\left(K_{b} b_{e q}+G_{R} R_{1} R_{2} K_{b} K_{p L}\right)}{J_{m} J_{L}} \\
d_{0} & =\frac{G_{R} R_{1} R_{2} K_{b} K_{i L}}{J_{m} J_{L}} .
\end{aligned}
$$

Singular perturbation analysis pertaining to this control scheme results in the following slow and fast characteristic polynomials:

$$
\begin{aligned}
& \psi_{l s}(s, \varepsilon) \approx s^{2}+\beta_{1} s+\beta_{0} \\
& \psi_{l f}(p, \varepsilon) \approx p^{2}-\beta_{1}^{\prime} p+\beta_{0}^{\prime}
\end{aligned}
$$

where

$$
\begin{aligned}
& \beta_{1}=\frac{G_{R}^{2} R_{2}^{2} b_{m}+R_{1}^{2} b_{L}+G_{R} R_{2} R_{1} K_{p L}}{G_{R}^{2} R_{2}^{2} J_{m}+R_{1}^{2} J_{L}}, \\
& \beta_{0}=\frac{G_{R} R_{2} R_{1} K_{i L}}{G_{R}^{2} R_{2}^{2} J_{m}+R_{1}^{2} J_{L}}, \\
& \beta_{1}^{\prime}=\frac{G_{R}^{2} R_{2}^{2} b_{m}+R_{1}^{2} b_{L}+G_{R}^{2} R_{2}^{2} K_{p L}}{G_{R}^{2} R_{2}^{2} J_{m}+R_{1}^{2} J_{L}} \varepsilon, \\
& \beta_{0}^{\prime}=\frac{G_{R}^{2} R_{2}^{2} J_{L}+R_{1}^{2} J_{m}}{J_{m} J_{L}} .
\end{aligned}
$$

Note that the slow subsystems are stable for all $K_{p L}, K_{i L}>0$. However, the fast subsystem is unstable for all $K_{p L}>0$ and $K_{i L}>0$. Also, note that the characteristic polynomials given by equations $(12 \mathrm{~b})$ and $(17 \mathrm{~b})$ are identical when $\varepsilon=0$. Thus, analyzing the limiting case of an infinitely stiff belt, that is, $\varepsilon=0$ will not reveal the instability exhibited by (17b).

Remark 1 Equation (1b) may be used to give an interpretation of the foregoing analysis. Differentiating (1b), we obtain

$$
J_{L} \ddot{\omega}_{L}+b_{L} \dot{\omega}_{L}+R_{2}^{2} K_{b} \omega_{L}=R_{1} R_{2} K_{b} \omega_{m}
$$

which indicates that $\omega_{L}$ can attain steady-state only when $\omega_{m}$ attains steadystate first. Even after $\omega_{m}$ attains steady-state, $\omega_{L}$ continues to exhibit damped oscillations. Thus, by measuring only $\omega_{L}$ and using the control law given by the (14), it will be difficult to distinguish whether the oscillations in $\omega_{L}$ are due to fluctuations in motor speed or, the oscillations are indeed damped oscillations. In such a situation, the controller attempts to react to the damped oscillations 
also, and in this process, changes $\omega_{m}$, which in turn affects $\omega_{L}$ because of the dynamics given by the (19). Thus, the control law given by (14) does not present a desirable situation.

\section{Simultaneous Motor and Load Speed Feedback Control Scheme}

In this scheme, the load speed control corrects directly the torque input to the system as shown in Figure 5. The closed-loop transfer function from $\omega_{r L}$

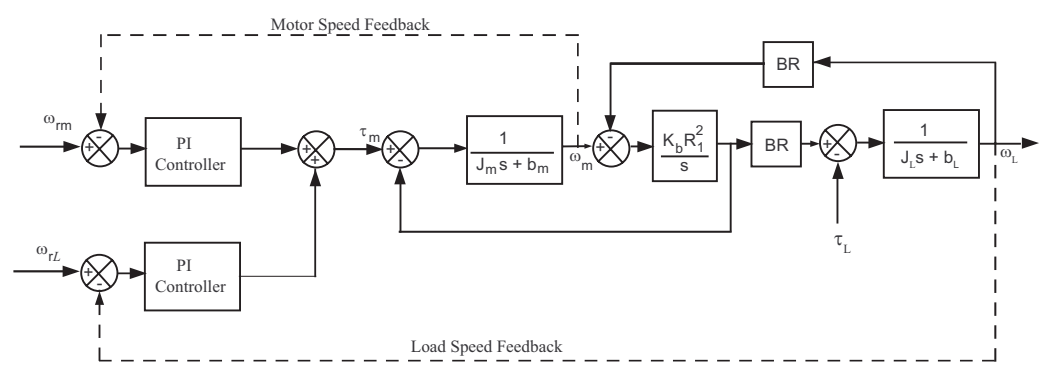

Fig. 5. Simultaneous motor and load speed feedback scheme: Torque mode

to $\omega_{L}$ is given by

where

$$
\frac{\omega_{L}(s)}{\omega_{r L}(s)}=\frac{\alpha_{m L t}}{\psi_{m L t}(s)}
$$

$$
\begin{gathered}
\alpha_{m L t}(s)=a_{1} s+a_{0} \\
a_{1}=\frac{\left(G_{R} R_{1} R_{2} K_{b} K_{p L}+G_{r} R_{2} K_{p m} / R_{1}\right)}{J_{m} J_{L}}, \\
a_{0}=\frac{\left(G_{R} R_{1} R_{2} K_{b} K_{i L}+G_{r} R_{2} K_{i m} / R_{1}\right)}{J_{m} J_{L}} \\
\psi_{m L t}(s)=s^{4}+f_{3} s^{3}+f_{2} s^{2}+f_{1} s+f_{0} \\
f_{3}=\frac{\left(b_{m} J_{L}+J_{m} b_{L}+J_{L} K_{p m}\right)}{J_{m} J_{L}} \\
f_{2}=\frac{\left(K_{b} J_{e q}+b_{m} b_{L}+K_{p m} b_{L}+J_{L} K_{i m}\right)}{J_{m} J_{L}}, \\
f_{1}=\frac{\left(K_{b} b_{e q}+G_{R}^{2} K_{b} K_{p m}+K_{i m} b_{L}+G_{R} R_{1} R_{2} K_{b} K_{p L}\right)}{J_{m} J_{L}} \\
f_{0}=\frac{K_{i m} G_{R}^{2} R_{2}^{2} K_{b}+G_{R} R_{1} R_{2} K_{b} K_{i L}}{J_{m} J_{L}} .
\end{gathered}
$$

Note that the coefficients $f_{0}$ to $f_{3}$ depend on the gains of the control law. Singular perturbation analysis for this case results in the slow and fast characteristic polynomials as

$$
\begin{aligned}
\psi_{m l s}(s, \varepsilon) & \approx s^{2}+\gamma_{1} s+\gamma_{0} \\
\psi_{m l f}(p, \varepsilon) & \approx p^{2}+\gamma_{1}^{\prime} p+\gamma_{0}^{\prime}
\end{aligned}
$$


where

$$
\begin{aligned}
\gamma_{1} & =\frac{G_{R}^{2} R_{2}^{2} b_{m}+R_{1}^{2} b_{L}+G_{R} R_{2} R_{1} K_{p L}+G_{R}^{2} R_{2}^{2} K_{p m}}{G_{R}^{2} R_{2}^{2} J_{m}+R_{1}^{2} J_{L}} \\
\gamma_{0} & =\frac{G_{R}^{2} R_{2}^{2} K_{i m}+G_{R} R_{2} R_{1} K_{i L}}{G_{R}^{2} R_{2}^{2} J_{m}+R_{1}^{2} J_{L}} \\
\gamma_{1}^{\prime} & =\frac{G_{R}^{2} R_{2}^{2} b_{m}+R_{1}^{2} b_{L}+G_{R}^{2} R_{2}^{2} K_{p L}+G_{R}^{2} R_{2}^{2} K_{p m}\left(J_{L} / J_{m}\right)}{G_{R}^{2} R_{2}^{2} J_{m}+R_{1}^{2} J_{L}} \varepsilon \\
\gamma_{0}^{\prime} & =\frac{G_{R}^{2} R_{2}^{2} J_{L}+R_{1}^{2} J_{m}}{J_{m} J_{L}} .
\end{aligned}
$$

Therefore, the slow and fast subsystems are stable for all positive controller gains. Note that the outputs of both load speed and motor speed controller combine to form a torque input to the motor; this is typically referred to as the torque mode in practice when multiple loops such as this are employed. Another strategy is to use the output of the load speed controller as the motor speed reference correction; a block diagram of such a scheme is provided in Fig. 11 in Appendix B. This strategy results in an unstable system which is shown in Appendix B.

\section{Adaptive Feedforward (AFF) Compensation to Reject Load Dis- turbances}

The use of feedforward compensation to reject known disturbances by direct cancelation or unknown disturbances by their estimation has been known to be effective in attenuating disturbances. We consider the rejection of periodic disturbances on the load by using an adaptive feedforward action based on load speed error. The control scheme that utilizes the feedforward action is shown in Figure 6. We use an adaptive feedforward algorithm given in [21] that

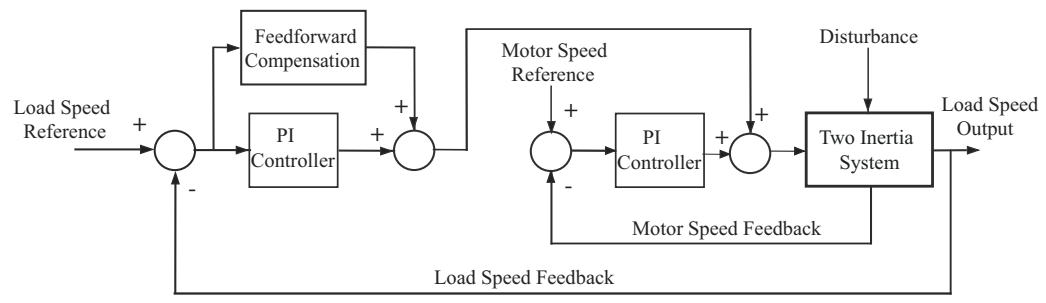

Fig. 6. Control scheme with feedback and feedforward compensation

is particularly applicable in this situation as the feedforward action preserves the stability of the overall system with the feedback controller with simultaneous motor and load speed feedback. The approach is briefly discussed as applicable to this problem; the details are given in [21]. The idea is to estimate the amplitude and phase of the disturbance for a known frequency of 
the disturbance. The disturbance can be expressed in the form

$$
\begin{aligned}
d & =\theta_{1}^{*} \cos (\omega t)+\theta_{2}^{*} \sin (\omega t) \\
& :=\phi(t) w_{0}^{*}
\end{aligned}
$$

where $\omega$ is a known frequency, $\theta_{1}^{*}$ and $\theta_{2}^{*}$ are unknown parameters. The adaptation laws for the unknown parameters $\theta_{1}^{*}$ and $\theta_{2}^{*}$ are given by the following simple pseudo-gradient algorithm:

$$
\begin{aligned}
& \dot{\theta}_{1}=\gamma e(t) \cos (\omega t), \\
& \dot{\theta}_{2}=\gamma e(t) \sin (\omega t),
\end{aligned}
$$

where $\theta_{1}$ and $\theta_{1}$ are the parameter estimates, $e(t)=\omega_{r L}-\omega_{L}$ is the load speed error, and $\gamma$ is the adaptation gain. Using the estimated parameters, the feedforward control action is given by

$$
u_{f}=-\theta_{1} \cos (\omega t)-\theta_{2} \sin (\omega t) .
$$

The estimation of the disturbance and its cancelation when the load speed error contains a sinusoidal component with frequency $\omega$ may be intuitively explained as follows. If the load speed error is $e(t)=\bar{e}(t)+e_{\theta_{1}} \sin (\omega t)$, in the adaptive algorithm the product $e(t) \sin (\omega t)$ will generate a positive $e_{\theta_{1}} \sin ^{2}(\omega t)$ term. This will result in a parameter drift which results in the attenuation of disturbance until it reaches its nominal value. At this point the load speed error is free of the sinusoidal component as the disturbance is compensated by feedforward control $u_{f}$. With the compensation, the product term $e(t) \sin (\omega t)$ in the parameter adaptive law becomes zero and the parameters converge. Since the regressor vector $\phi(t)$ is persistently exciting, the parameter vectors converge to zero. In fact, this adaptive feedforward action with estimation of disturbance parameters using the pseudo-gradient algorithm has been shown to be equivalent to the use of the internal model of the disturbance in [21].

\section{Experiments}

A picture of experimental setup is shown in Figure 7. It consists of an $\mathrm{AC}$ motor shaft connected to the load shaft (roll) via a belt-pulley and gear-pair transmission. A $15 \mathrm{HP}(11.19 \mathrm{KW})$ AC motor with a rated speed of 1750 $\mathrm{RPM}$ is employed. The belt ratio $\left(B R=\left(R_{2} / R_{1}\right) G_{R}\right)$ for the transmission is 3.825. An encoder on the motor shaft is employed to measure the motor shaft speed and a laser sensor is used to measure the load shaft speed. The real-time hardware, including the drives, controller, and communication network, was provided by Rockwell Automation (Allan-Bradley). All the real-time hardware components of the machine are connected through a ControlNet communication network. The network is updated every $5 \mathrm{~ms}$ (Network Update Time) and 

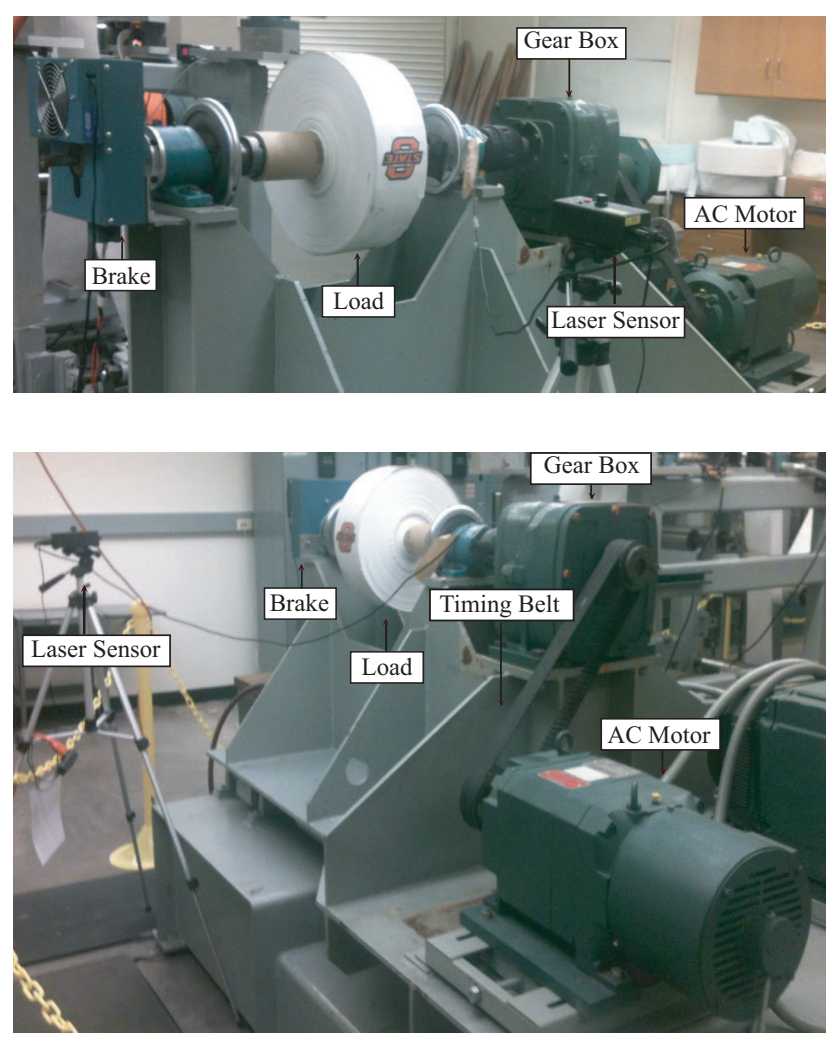

Fig. 7. Picture of the experimental platform. Top view: Load side. Bottom view: Motor side.

data is communicated to the network every $10 \mathrm{~ms}$ (Request Package Interval). A brake is attached on the other side of the load shaft to inject periodic torque disturbances; a magnetic clutch brake (Magpower GBC 90) that can apply 26 lb-ft torque is used.

7.1 Design and Implementation Guidelines for the Proposed Load Speed Regulation Scheme

The following guidelines should provide assistance to practicing engineers in the design and implementation of the proposed load speed regulation scheme.

(1) Select the PI gains for the motor speed PI controller by tuning them for the desired regulation performance without the load. These PI gains will provide a baseline for the motor speed PI controller.

(2) Select the PI gains for the load speed feedback PI controller by placing stable closed-loop poles for the fast and slow dynamics given in Section 5. It may be necessary to re-tune the PI gains of the motor speed PI controller to obtain the desired closed-loop poles.

(3) Adaptive feedforward compensation is generated based on the disturbance parameter estimates given in Section 6. Although some tuning of 
the adaptation gain $\gamma$ may provide better transient response, in theory any positive gain and reasonable initial conditions for the estimates would work. For simplicity, we have chosen the values of $\gamma=1$ and the initial conditions for both estimates to be zero; this approach has worked in a number of experiments we have conducted with different disturbances.

(4) Ensure that the the bandwidth of the drive system is sufficient enough to attenuate the targeted disturbance frequency.

(5) The adaptive feedforward compensation requires the knowledge of the disturbance frequency. This can be obtained in multiple ways. One can obtain the frequency spectrum of the load speed to determine all the disturbance frequencies in the frequency region of interest. It is possible to attenuate more than one frequency by simply cascading multiple adaptive feedforward compensation blocks with each targeting a particular frequency. In the case of a roll-to-roll system, the disturbance frequency may be simply obtained from the line speed and the radius of the roller.

The PI controller gains for the motor speed loop were chosen to be $K_{p m}=15$ and $K_{i m}=3.09$ and for the load speed loop to be $K_{p L}=0.07$ and $K_{i L}=0.001$. A number of experiments were conducted at different reference speeds to evaluate the performance of proposed control scheme. In each experiment, the brake provides an external periodic disturbance torque of the form $A+B \sin \left(\omega_{d} t\right)$ $(A=2, B=1.5)$. The following disturbance frequencies were injected to evaluate the control schemes: $\omega_{d}=0.05,0.15,0.25 \mathrm{~Hz}$. These disturbances are typical of the disturbances that are observed in roll-to-roll manufacturing machines where such transmission systems are typically employed. The adaptation gain $\gamma=1$ is chosen and the initial values of the estimates are set to zero.

Figure 8 shows the evolution of the load speed (reflected to the motor side) in the presence of disturbance with frequency $0.25 \mathrm{~Hz}$ when the reference speed is 719 RPM without the use of the adaptive feedforward action. Figure 9 shows the Fast Fourier Transform (FFT) of the load speed for the two cases. It is evident that the control scheme with the AFF action (shown in Figure 6) can provide significantly improved load speed regulation. Figure 10 control torque input corresponding to the two cases, without and with adaptive feedforward compensation. It is evident that the torque input is larger when the adaptive feedforward is employed. Table 1 shows the standard deviation of the load speed signal from its reference for the various schemes. It is clear that the employing load speed feedback in addition to motor speed feedback can improve performance. Further, use of the adaptive feedforward action based on load speed feedback can significantly improve the regulation performance. 

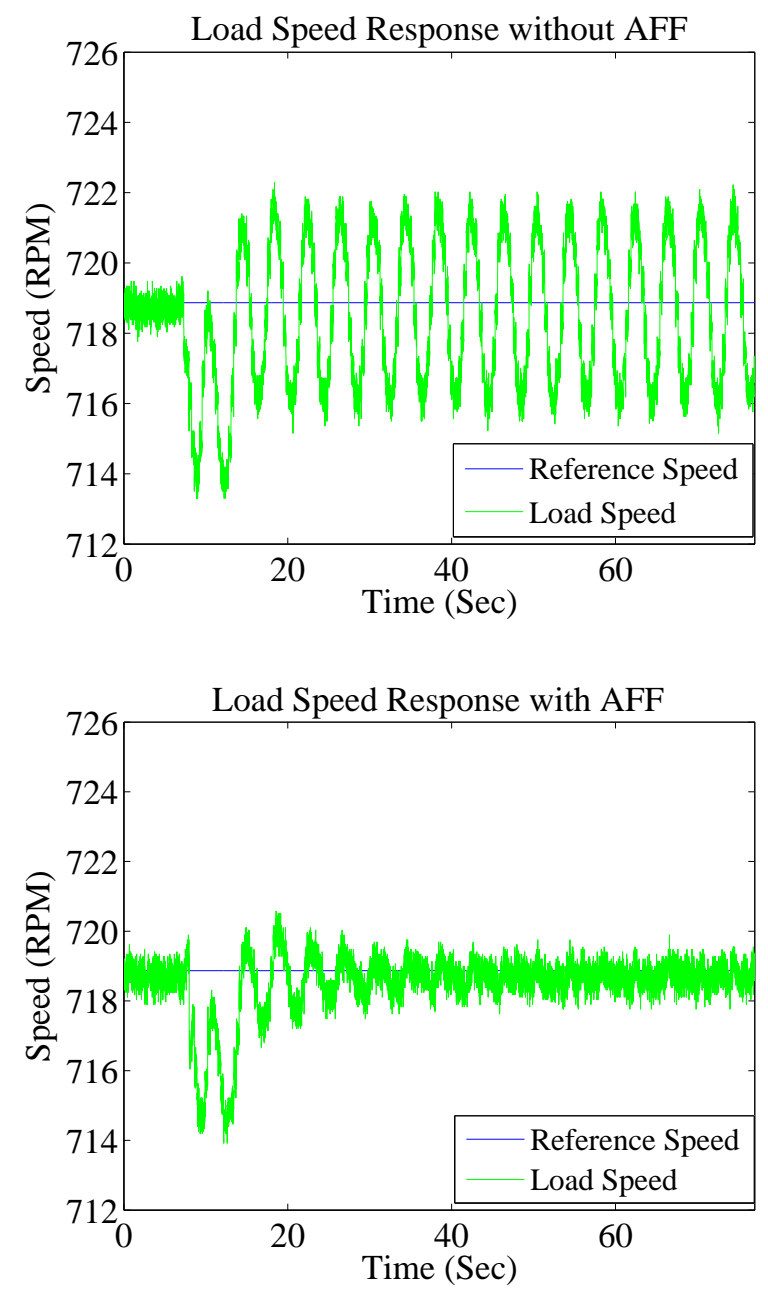

Fig. 8. Load speed response with $0.25 \mathrm{~Hz}$ torque disturbance. Top: Without AFF. Bottom: With AFF

Table 1

Comparison of different control schemes

\begin{tabular}{|c|c|c|c|}
\hline \multirow{2}{*}{$\begin{array}{c}\text { Disturbance } \\
\text { Frequency }\end{array}$} & \multicolumn{3}{|c|}{ Standard Deviation } \\
\cline { 2 - 4 } & Only Motor & $\begin{array}{c}\text { Motor + Load } \\
\text { Feedback }\end{array}$ & $\begin{array}{c}\text { Motor + Load } \\
\text { Feedback + AFF }\end{array}$ \\
\hline $0.25 \mathrm{~Hz}$ & 2.09 & 1.35 & 0.34 \\
\hline $0.15 \mathrm{~Hz}$ & 4.71 & 3.53 & 0.87 \\
\hline $0.05 \mathrm{~Hz}$ & 3.89 & 2.47 & 0.68 \\
\hline
\end{tabular}

\section{Conclusions}

We have investigated the problem of regulating load speed in a mechanical transmission with a compliant belt. Several speed control strategies that rely 


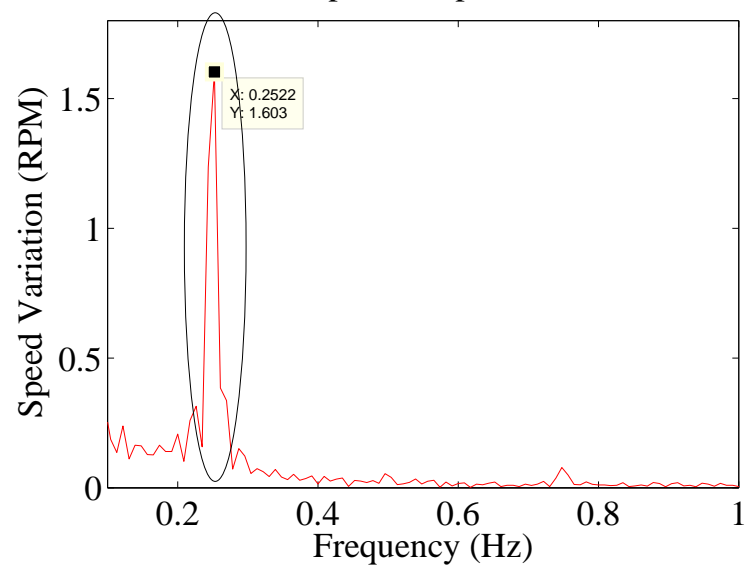

FFT of Load Speed Response with AFF

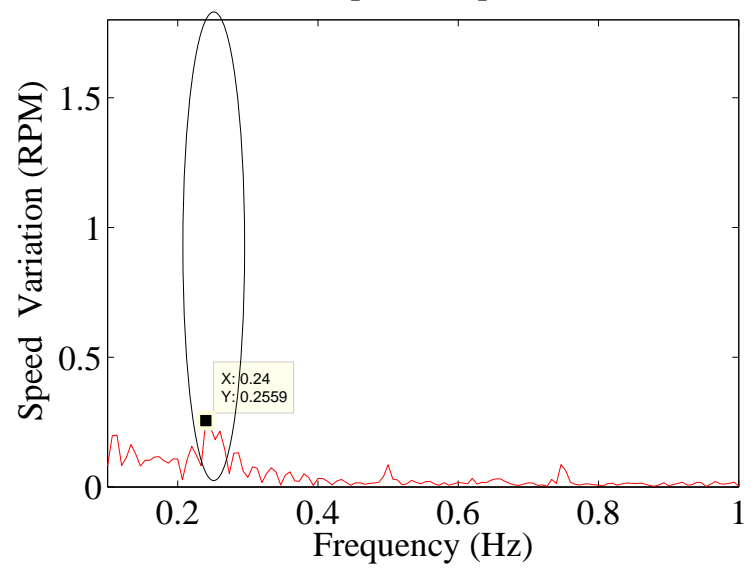

Fig. 9. FFT of load speed response with and without AFF

on either motor speed feedback or load speed feedback or both are investigated. A singular perturbation approach with the inverse of the belt compliance as the small parameter is employed to analyze different control strategies. It is shown that the system is unstable when pure load speed feedback is employed. A control system that considers both motor speed feedback and load speed feedback in the torque mode is stable and can provide improved closed-loop performance. Since the feedback control action is not sufficient to reject periodic load disturbances, an adaptive feedforward algorithm is designed to estimate the disturbance and generate a compensation term to attenuate periodic disturbances of known frequency and unknown amplitude. Experiments were conducted on an industrial grade transmission system to evaluate the control schemes and compare their performance. Although we have used only belt compliance as the compliant element in the transmission system, torsional compliance due to long shafts can also be included and the analysis conclusions will remain the same. 

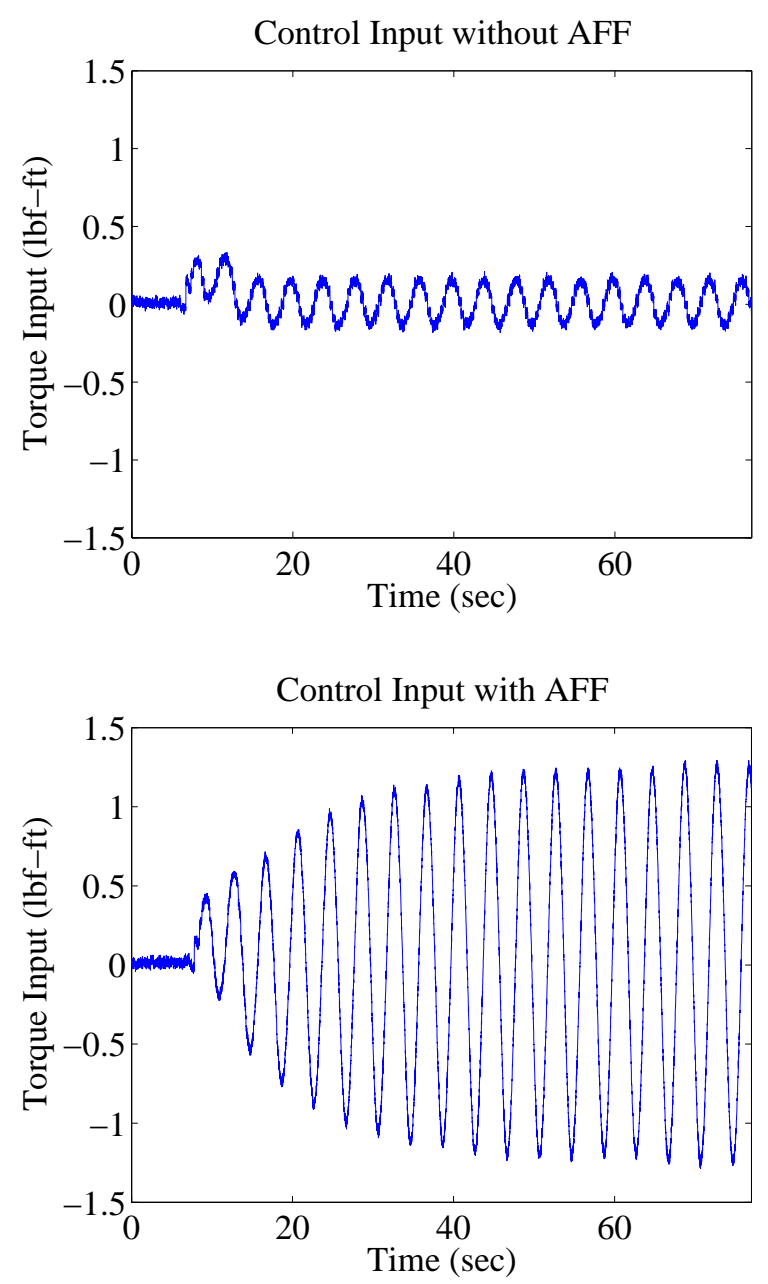

Fig. 10. Control input with $0.25 \mathrm{~Hz}$ torque disturbance. Top: Without AFF. Bottom: With AFF

\section{Acknowledgement}

This work was supported by the Web Handling Research Center at Oklahoma State University and the US National Science Foundation.

\section{References}

[1] P. R. Pagilla and Y. Diao, "Resonant frequencies in web process lines due to idle rollers and spans," ASME Journal of Dynamic Systems, Measurement and Control, vol. 133, no. 6, November 2011.

[2] T. C. Firbank, "Mechanics of the belt drive," International Journal of Mechanical Sciences, vol. 12, pp. 1053-1063, 1970. 
[3] J. N. Fawcett, "Chain and belt drives - a review," Shock and Vibration Digest, vol. 13 (5), pp. 5-12, 1981.

[4] W. T. Townsend and J. K. Salisbury, "The efficiency limit of belt and cable drives," ASME Journal of Mechanisms, Transmissions and Automation in Design, vol. 110, pp. 303-307, September 1988.

[5] W. J. Book, "Controlled motion in an elastic world," ASME Journal of Dynamic Systems, Measurement, and Control, vol. 115, pp. 252-261, June 1993.

[6] S. J. Hwang, N. C. Perkins, A. G. Ulsoy, and R. J. Meckstroth, "Rotational response and slip prediction of serpentine belt drive systems," ASME Journal of Vibration and Acoustics, vol. 116, pp. 71-78, January 1994.

[7] G. Gerbert, "Belt slip - a unified approach," ASME Journal of Mechanical Design, vol. 118, pp. 432-438, September 1996.

[8] M. Corporation, "Timing belt theory," Tech. rep., Mectrol Corporation, 2002.

[9] M. Yoshida, H. Ishida, M. Takahashi, and I. Kasai, "An analysis of belt transfer process," Tech. Rep. 0916-8087, Japan Hardcopy, pp. 185-188, 1997.

[10] M. J. Leamy, J. R. Barber, and N. C. Perkins, "Dynamics of belt/pulley frictional contact," Journal of Solid Mechanics and its applications, vol. 72, pp. 277-286, 1999.

[11] S. E. Bechtel, S. Vohra, K. I. Jacob, and C. D. Carlson, "The stretching and slipping of belts and fibers on pulleys," ASME Journal of Applied Mechanics, vol. 67, pp. 197-206, March 2000.

[12] W. Li and M. Rehani, "Modeling and control of a belt-drive positioning table," Proceedings of the 22nd International Conference on Industrial Electronics, Control, and Instrumentation, vol. 3, pp. 1984-1989, August 1996.

[13] J. F. Pan, N. C. Cheung, and Y. Zou, "High-precision control of LSRM based x-y table for industrial applications," ISA Transactions, vol. 52, pp. 105-114, 2013.

[14] M. EI-Sharkawi and Y. Guo, "Adative fuzzy control of a belt-driven precision positioning table," in In Proceedings of the IEEE International Electric Machines and Drives Conference, vol. 3, June 2003, pp. 1504-1506.

[15] A. Hace, K. Jezernik, and M. Terbuc, "Robust motion control algorithm for belt-driven servomechanism," in In Proceedings of the IEEE International Symposium on Industrial Electronics, Bled, Slovenia, 1999, pp. 893-898.

[16] R. V. Dwivedula and P. R. Pagilla, "Effect of backlash on web tension in roll-to-roll manufacturing systems: Mathematical model, mitigation method and experimental evaluation," IEEE Multi-conference on Systems and Control, February 2013.

[17] R. Dhaouadi, K. Kubo, and M. Tobise, "Two-degree-of-freedom robust speed controller for high-performance rolling mill drives," IEEE Transactions on Industry Applications, vol. 29 (5), 1993. 
[18] G. Bradenburg and U. Schafer, "Design and performance of different types of observers for industrial speed and position controlled electromechanical systems," in Proc. Int. Conf. of Electrical Drives and Power Electronics, Slovakia, 1990, pp. 1-10.

[19] S. Colombi and T. Raimondi, "Compliance compensation in mechatronic systems," 20th International Conference on Industrial Electronics, Control and Instrumentation, vol. 2, pp. 946-951, September 1994.

[20] Y. S. Lee and K. C. Hsu, "Shaft torsional oscillation of induction machine including saturation and hysteresis of magnetizing branch with an inertia load," in In Proceedings of International Conference on Energy, Management and Power Delivery, vol. 1, November 1995, pp. 134-139.

[21] M. Bodson, "Rejection of periodic disturbances of unknown and time-varying frequency," International Journal of Adaptive Control and Signal Processing, vol. 19, pp. 67-88, 2005.

[22] P. V. Kokotovic, H. K. Khalil, and J. O'Reilly, Singular Perturbation Methods in Control: Analysis and Design, 1st ed., ser. Society for Industrial and Applied Mathematics edition. Academic Press, London, 1986.

[23] S. Dubowsky and F. Freudenstein, "Dynamic analysis of mechanical systems with clearance: Part 1: Formulation of dynamic model," ASME Journal of Engineering for Industry, vol. 93, pp. 305-309, February 1971.

\section{Appendix}

\subsection{Stability of the System with only Motor Speed Feedback}

The following theorem provides the stability of the system with only motor feedback that is discussed in Section 3.

Theorem 2 The closed-loop system defined by (4) and (1) is stable and $\omega_{m}$ approaches $\omega_{r m}$ for all $K_{p m}, K_{i m}>0$.

Proof: Substituting (4) into (1) and neglecting backlash effect, we obtain dynamics of the closed-loop system as

$$
\begin{gathered}
K_{p m}\left(\omega_{r m}-\omega_{m}\right)+K_{i m} \int\left(\omega_{r m}-\omega_{m}\right) d \tau=\left(J_{m} \ddot{\theta}_{m}+b_{m} \dot{\theta}_{m}\right) \\
+R_{1} K_{b}\left(R_{1} \theta_{m}-G_{R} R_{2} \theta_{L}\right), \\
G_{R} R_{2} K_{b}\left(R_{1} \theta_{m}-G_{R} R_{2} \theta_{L}\right)=\left(J_{L} \ddot{\theta}_{L}+b_{L} \dot{\theta}_{L}\right) .
\end{gathered}
$$


Differentiate (28) to obtain

$$
\begin{array}{r}
-K_{p m} \dot{\omega}_{m}+K_{i m}\left(\omega_{r m}-\omega_{m}\right)=\left(J_{m} \ddot{\omega}_{m}+b_{m} \dot{\omega}_{m}\right) \\
+R_{1} K_{b}\left(R_{1} \omega_{m}-G_{R} R_{2} \omega_{L}\right), \\
G_{R} R_{2} K_{b}\left(R_{1} \omega_{m}-G_{R} R_{2} \omega_{L}\right)=\left(J_{L} \ddot{\omega}_{L}+b_{L} \dot{\omega}_{L}\right) .
\end{array}
$$

Defining errors, $e_{m}=\omega_{m}-\omega_{r m}$ and $e_{L}=\omega_{L}-\left(R_{1} / G_{R} R_{2}\right) \omega_{r m},(29)$ may be written as

$$
\begin{array}{r}
-K_{p m} \dot{e}_{m}-K_{i m} e_{m}=J_{m} \ddot{e}_{m}+b_{m} \dot{e}_{m} \\
+R_{1} K_{b}\left(R_{1} e_{m}-G_{R} R_{2} e_{L}\right), \\
G_{R} R_{2} K_{b}\left(R_{1} e_{m}-G_{R} R_{2} e_{L}\right)=J_{L} \ddot{e}_{L}+b_{L} \dot{e}_{L}
\end{array}
$$

Choose

$$
\begin{aligned}
V(t) & =\frac{1}{2}\left(J_{m} \dot{e}_{m}^{2}+J_{L} \dot{e}_{L}^{2}+K_{b}\left(R_{1} e_{m}-G_{R} R_{2} e_{L}\right)^{2}\right. \\
& \left.+K_{i m} e_{m}^{2}\right) .
\end{aligned}
$$

Then, the time derivative of $V$ along the trajectories defined by (30) is obtained to be

$$
\frac{d V(t)}{d t}=-\left(b_{m}+K_{p m}\right) \dot{e}_{m}^{2}-b_{L} \dot{e}_{L}^{2}
$$

Thus, $V(t)$ is a Lyapunov function and $e_{m}, e_{L}, \dot{e}_{m}, \dot{e}_{L} \in \mathcal{L}_{\infty}$ which implies, from (30), that $\ddot{e}_{m}, \ddot{e}_{L} \in \mathcal{L}_{\infty}$. From (31) and (32), we conclude that because $V(t)$ is bounded from below and is nonincreasing with time, it has a limit, i.e., $\lim _{t \rightarrow \infty} V(t)=V_{\infty}$. Now from (32), we have

$$
\lim _{t \rightarrow \infty} \int_{0}^{t}\left(b_{m}+K_{p m}\right) \dot{e}_{m}^{2}+b_{L} \dot{e}_{L}^{2}=V_{0}-V_{\infty}<\infty
$$

Therefore, $\dot{e}_{m}, \dot{e}_{L} \in \mathcal{L}_{2}$ and by Barbalat's Lemma, we have $\dot{e}_{m} \rightarrow 0$ and $\dot{e}_{L} \rightarrow 0$. Thus, $\omega_{m}$ and $\omega_{L}$ tend to become constants as $t \rightarrow \infty$ and from (29), we see that $\omega_{m} \rightarrow \omega_{r m}$ and $\omega_{L} \rightarrow\left(R_{1} / G_{R} R_{2}\right) \omega_{r m}$. 


\subsection{Speed Correction Based Simultaneous Motor and Load Speed Feedback Control Scheme}

The control scheme that utilizes both motor and load speed feedback discussed in Section 5 considers the output of the load speed and motor speed controllers as torque correction. There is also another control scheme that is employed in practice where the outer load speed loop provides a speed reference correction to the inner motor speed which is shown in Fig. 11. In the following we show that such a control scheme results in an unstable system, and thus must be avoided. For this analysis, we employ a simple proportional control action for the load speed controller and a PI controller for the motor speed loop. The

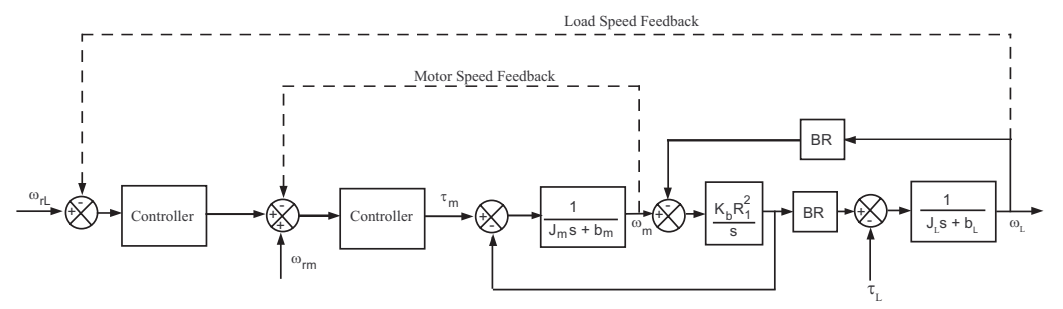

Fig. 11. Simultaneous motor and load speed feedback scheme: Speed mode

closed-loop transfer function from $\omega_{r L}$ to $\omega_{L}$ for this strategy is obtained as

$$
\frac{\omega_{L}(s)}{\omega_{r L}(s)}=\frac{\left(G_{R} R_{1} R_{2} K_{b} / J_{m} J_{L}\right) \alpha_{m L s}}{\psi_{m L s}(s)}
$$

where

$$
\begin{aligned}
& \alpha_{m L s}(s)=K_{p m} K_{p L} s+K_{i m} K_{p L} \\
& \psi_{m L s}(s)=s^{4}+e_{3} s^{3}+e_{2} s^{2}+e_{1} s+e_{0}, \\
& e_{3}=\frac{\left(b_{m} J_{L}+J_{m} b_{L}+J_{L} K_{p m}\right)}{J_{m} J_{L}}, \\
& e_{2}=\frac{\left(K_{b} J_{e q}+b_{m} b_{L}+b_{L} K_{p m}+J_{L} K_{i m}\right)}{J_{m} J_{L}}, \\
& e_{1}=\frac{\left(K_{b} b_{e q}+G_{R}^{2} R_{2}^{2} K_{b} K_{p m}+b_{L} K_{i m}\right)}{J_{m} J_{L}} \\
& +\frac{\left(G_{R} R_{1} R_{2} K_{b} K_{p m} K_{p L}\right)}{J_{m} J_{L}}, \\
& e_{0}=\frac{\left(G_{R}^{2} R_{2}^{2} K_{b} K_{i m}+G_{R} R_{1} R_{2} K_{b} K_{i m} K_{p L}\right)}{J_{m} J_{L}} .
\end{aligned}
$$


Singular perturbation analysis results in the following slow and fast characteristic polynomials:

$$
\begin{aligned}
& \psi_{l s}(s, \varepsilon) \approx s^{2}+\delta_{1} s+\delta_{0} \\
& \psi_{l f}(p, \varepsilon) \approx p^{2}-\delta_{1}^{\prime} p+\delta_{0}^{\prime}
\end{aligned}
$$

where

$$
\begin{aligned}
& \delta_{1}=\frac{G_{R}^{2} R_{2}^{2} b_{m}+R_{1}^{2} b_{L}+G_{R}^{2} R_{2}^{2} K_{p m}+\left(G_{R}^{2} R_{2}^{2} / R_{1}\right) K_{p m} K_{p L}}{G_{R}^{2} R_{2}^{2} J_{m}+R_{1}^{2} J_{L}} \\
& \delta_{0}=\frac{G_{R}^{2} R_{2}^{2} K_{i m}+\left(G_{R}^{2} R_{2}^{2} / R_{1}\right) K_{i m} K_{p L}}{G_{R}^{2} R_{2}^{2} J_{m}+R_{1}^{2} J_{L}} \\
& \delta_{1}^{\prime}=\frac{G_{R}^{2} R_{2}^{2} b_{m}+R_{1}^{2} b_{L}+G_{R}^{2} R_{2}^{2} K_{p L} K_{p m}+G_{R}^{2} R_{2}^{2} K_{p m}\left(J_{L} / J_{m}\right)}{G_{R}^{2} R_{2}^{2} J_{m}+R_{1}^{2} J_{L}} \varepsilon \\
& \delta_{0}^{\prime}=\frac{G_{R}^{2} R_{2}^{2} J_{L}+R_{1}^{2} J_{m}}{J_{m} J_{L}} .
\end{aligned}
$$

Note that the slow subsystem is stable for all $K_{p m}, K_{i m}$, and $K_{p L}$. However, the fast subsystem is unstable for all $K_{p L}>0$. The instability of the system is also evident from simple root locus analysis of the closed-loop characteristic with varying $K_{p L}$, which is shown in Figure 12 .

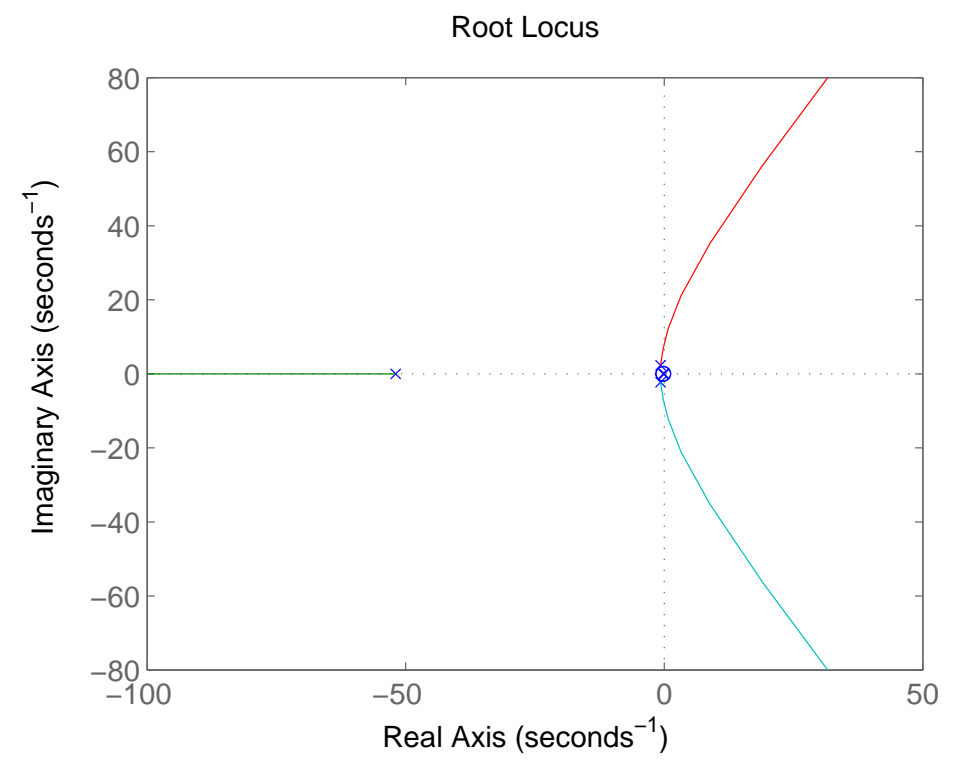

Fig. 12. Root locus plot with varying $K_{p L}$ in speed mode 